\title{
Die württembergischen Liberalen am Vorabend der Revolution von 1848
}

\author{
Von Hans Peter Müller
}

\section{Württemberg im Zeichen politischer, sozialer und ökonomischer Gärung}

Spätestens seit der Mitte der 1840er Jahre befand sich ganz Deutschland und so auch das Königreich Württemberg in einem alle Lebensbereiche umfassenden $\mathrm{Zu}$ stand der "Gärung“, der als Charakterisierung sowohl in den zeitgenössischen Quellen ${ }^{1}$ als auch in der modernen Historiographie immer wieder auftaucht ${ }^{2}$.

Auf politischem Gebiet war vor allem das Besitz- und Bildungsbürgertum mit dem den Deutschen Bund beherrschenden antiliberalen System Metternichs unzufrieden, das ein zorniger Zeitgenosse treffend als „kleinliche polizeiliche Staatskunst, die dreißig Jahre lang alle inneren Verhältnisse beherrscht hat, die unwürdige Bevormundung im Großen und Kleinen, die Präventivpolizei sammt der ganz byzantinisch ausgedüftelten Wissenschaft der Verbote, Hemmungen, Schranken und Chikanen“ charakterisierte ${ }^{3}$. Dieser Personenkreis hatte sich im Vormärz in den Kammern der süddeutschen Verfassungsstaaten als auf Reformen drängende liberale Opposition etabliert - eine Opposition allerdings ohne Organisation, die so letztlich kein gleichwertiger Gegner der Regierenden war.

1 Vgl. etwa „Beobachter“ (künftig: Beob.) Nr. 25 vom 26.1.1847 (dort in zeitgenössischer Schreibweise Gährung). Der Gaildorfer Glasfabrikant Gottlieb Rau sprach von einer krankhafte[n] Sehnsucht nach einem unbekannten Etwas, das beinahe alle Bevölkerungskreise umfasse, Beob. Nr. 99 vom 14.4.1846.

2 Vgl. etwa Rudolf Stadelmann, Soziale und politische Geschichte der Revolution von 1848, Darmstadt 1962, S.5. Er betont die vielen „Symptome“, die „die Ahnung von der Unausweichlichkeit einer Katastrophe“ bewirkten; ebd., S.17.

${ }^{3}$ Ludwig Häusser, Denkwürdigkeiten zur Geschichte der Badischen Revolution, Heidelberg 1851, S.5. Demzufolge sei der Unglaube an die Daner der bestehenden Zustände schon lange vor der Revolution Allgemeingut gewesen; ebd., S. 8. 
Ihre Gedankenwelt fußte auf den Ideen von 1789 und der französischen Publizistik; man studierte und bewunderte das staatliche Leben Englands und der USA und orientierte sich konkret am Vorbild der Schweiz 4 . Handlungsmaximen bot das vielbändige, seit den 1830 er Jahren erscheinende Staatslexikon der Freiburger Professoren Rotteck und Welcker. Dort konnte man „das Verfassungswesen der großen Völker studieren und in schmerzlicher Scham mit der deutschen Lage vergleichen“5. Das Lexikon galt als „Grundbuch des vormärzlichen Liberalismus vornehmlich in Süddeutschland " 6 - die beiden Professoren wurden als Akteure im badischen Landtag deutschlandweit bewundert. Für Württemberg galten zudem das von Robert Mohl 1829 bearbeitete Staatsrecht und seine Polizeiwissenschaft von 1833 als eine Art Blaupause liberalen Wollens ${ }^{7}$.

Es waren wie angedeutet keineswegs nur die politischen Verhältnisse, die weitgehende Unzufriedenheit und Unsicherheit in Württemberg hervorriefen. Gottlieb Rau (1816-1854) wurde am Vorabend der Revolution wohl zum schärfsten und unermüdlichsten Kritiker und Kronzeugen der sozialen und ökonomischen Verhältnisse, der zahlreiche Forderungen artikulierte. Im April 1846 konstatierte er eine zunehmende Kraftlosigkeit sowie eine Krankheitserscheinung in vielen Landesteilen, ablesbar an sinkende [r] Erwerbsfäbigkeit sowie zunebmende $[\mathrm{r}] \mathrm{Zah}$ lungsunfähigkeit und resultierend in Armut und Elend. Kritisch merkte er an, dass es nur an einem kräftigen allgemeinen Wollen fehle, um ein Vorwärts zu bewirken ${ }^{8}$. Er forderte etwa eine Nationalbank und ein Handelsministerium ${ }^{9}$, sprach sich für die Errichtung von Fabriken als Beschäftigungsanstalten aus ${ }^{10}$, warnte aber vor dem Irrglauben des Freihandels und der zermalmende[n] Gewalt des ausländischen Kapitals ${ }^{11}$. Für sein soziales Gewissen sprach ein Aufruf zu Gunsten der zurückkehrenden oder noch in Ungarn und Siebenbürgen verbliebenen unglücklichen Auswanderer. Für sie gelte es Hilfe zu schaffen durch Gründung nener Gewerbsanstalten ${ }^{12}$.

${ }^{4}$ Ausführlich dazu Franz Schnabel, Deutsche Geschichte im neunzehnten Jahrhundert, Monarchie und Volkssouveränität (Herder Taschenbuch, Bd.205), Freiburg 1964, S. $230-245$.

5 Theodor Heuss, 1848 - Werk und Erbe, Stuttgart 1948, S. 51.

${ }^{6}$ Schnabel (wie Anm. 4) S. 223.

7 In seinem „Staatsrecht des Königreiches Württemberg, 1. Teil (Verfassungsrecht), Tübingen 1829“ betonte Mohl im Vorwort, es gelte „noch viele Lücken in dem Gebäude der constitutionellen Monarchie auszufüllen“ und dieses „nach dem Bedürfnisse der neueren Zeit und Verfassung zu ändern“. Er habe sich nicht auf eine „bemerkungslose“ Behandlung beschränkt, sondern auch Lob und Tadel ausgesprochen; ebd., S. VIf.

8 Beob. Nr. 99 vom 14.4.1846 (z.T. gesperrt).

9 Beob. Nr.6 vom 8.1.1846.

10 Beob. Nr. 56 vom 27.2.1846, Nr.74 vom 17.3.1846 und Nr. 134 vom 18.5.1846.

11 Beob. Nr. 89 vom 1.4.1846.

12 Beob. Nr. 159 vom 14.6.1846. 
Zu Beginn des Jahres 1847 übergab Rau dem Landtag eine Denkschrift mit dem Titel Der Zustand des Landes wie er war, wie er ist und wie er seyn sollte. Dort sah er etwa in der dramatisch steigenden Auswanderung ein Krisensymptom auch für Württemberg. Die Mitnahme von Kapital ins Ausland sei verderblich für die Bleibenden, trage bei zum Landesruin und verstärke das Uebel der Verarmung aller Stände. Im Zollverein sah er schädliche Einflüsse auf die heimischen Gewerbe und kritisierte die industriefeindliche Haltung des Staates, der eigentlich eine liebende Mutter für alle sein sollte. Verarmung und Hunger könne man nur bekämpfen, wenn überall Neues geschaffen werde. Das zu erstrebende Neue sah er 1. in einer Kolonisation großer Oedungen und Schaffung neuer Anbauflächen, verbunden mit dem Anbau von Obstbäumen und einem entsprechenden Export, 2. in einer Hebung der Gewerbeanstalten im Großen durch Staatsbeiträge, 3. in der Emporbringung des Aktivbandels besonders nach Nordamerika. Solche Maßnahmen zur Hebung der Produktivkräfte erschienen ihm wichtiger als der vieldiskutierte Eisenbahnbau ${ }^{13}$. Rau wirkte nicht nur als soziale Kassandra, er war auch zum politischen Engagement bereit und bewarb sich u. a. 1846 erfolglos um das Uracher Landtagsmandat. Den dortigen Wahlmännern präsentierte er eine Art Glaubensbekenntnis, in dem er wiederum die Ursache der sozialen und ökonomischen Misere benannte, zu ergreifende Maßnahmen formulierte und sich zudem zu den politischen Forderungen der Opposition bekannte ${ }^{14}$.

Im Januar 1847 konstatierte ein Artikel im Parteiblatt, die vorhandenen Uebelstände beschränkten sich keineswegs nur auf die Politik, vielmehr sei auch das bäusliche, soziale und kirchliche Leben an bundertfachen Schäden erkrankt ${ }^{15}$. Der „Beobachter“ sah es als seine Aufgabe, sich ganz im Sinne Raus zur Linderung des Nothstandes zu äußern. Er konstatierte eine weitgehende Erregung im Lande und beklagte insbesondere die allgemeine Teuerung ${ }^{16}$. Vor diesem Hintergrund artikulierte er die Hoffnung auf das Wirken des Landtags ${ }^{17}$.

Nachdem die allgemeine Krisensituation ihren unübersehbaren Ausdruck in Hungerkrawallen in Stuttgart und Ulm Anfang Mai $1847^{18}$ gefunden hatte, änderte und verschärfte sich die öffentliche Auseinandersetzung. Während König Wilhelm

13 Die Denkschrift nach den Berichten im Beob. Nr. 28, 29, 32 und 34 vom 29.1.1847 bis 4.2.1847. Die Zitate aus Nr.29, 32 und 34.

${ }^{14}$ Vgl. Beob. Nr. 213 vom 6.8.1846. - Vgl. zu ihm etwa Klaus Peter Eichele, Traum und Fiasko des Gottlieb Rau (1816-1854), Leben und Zeit des Revolutionärs und Glasfabrikanten aus Gaildorf, Tübingen 1991.

15 Beob. Nr. 25 vom 26.1.1847.

16 Die durch Missernten hervorgerufene Verteuerung der Lebensmittel seit 1846 ließ das Blatt zuvor die Furcht vor dem Wachsthum des Proletariates äußern. Beob. Nr.9 vom 10.1.1847.

17 Beob. Nr.76 vom 18.3.1847.

${ }_{18}$ Dazu und zu den Folgen ausführlich Dieter Langewiesche, Liberalismus und Demokratie in Württemberg zwischen Revolution und Reichsgründung, Düsseldorf 1974, S. 84-93. 
überzeugt war, dass Liberale wie Römer und Murschel „einen großen Aufstand“ geplant hätten ${ }^{19}$, sah sich der „Beobachter“ einem Platzregen gegnerischer Unterstellungen ausgesetzt, die darin kulminierten, dass man Sympathien für den Tumult gehegt, den Militäreinsatz missbilligt und so den Arm der gesetzmäßigen Gewalt [habe] lähmen wollen. In der Ulmer Chronik wurde behauptet, die Liberalen hätten auf den Schlossplatz ziehen wollen um Römer I. auszurufen. Die bisher besitzbürgerlich orientierten Liberalen pflegten nun die engsten Beziehungen mit dem Proletariate. Der „Beobachter“ konstatierte einen Waffenwechsel seiner verblendeten Gegner; die bisher verspottete Opposition werde jetzt des Umsturzes aller Dinge beschuldigt ${ }^{20}$.

In der Folgezeit stellte sich das Oppositionsblatt der Frage Wo feblt's uns? Angesichts entsetzliche $[\mathrm{r}]$ leibliche $[\mathrm{r}]$ und sitten- und ordnungslose[r] Armuth konstatierte man eine von weitreichender Unzufriedenheit und Missstimmung begleitete Krankheit in unserem gesellschaftlichen Wesen. Die bürgerliche Gesellschaft sei bedroht und sehe macht- und ratlos einem drobenden Sturme entgegen. Kritisch wurde angemerkt, dass u.a. Schlaffheit und Mangel an jeder Art von Gemeingeist, ein fehlendes Rechtsgefühl und Selbstsucht vorherrschten, obwohl die Staatsweisheit ein gemeinschaftliches Wirken sozusagen diktiere ${ }^{21}$.

Dieses Bekenntnis bürgerlicher Verunsicherung führte Ende Oktober zu einem bemerkenswerten Fazit des „Beobachters“: Die Erfahrung von Raub und Bestialität im Mai hätten klar werden lassen, dass zum Schutz gegen die communistische Gährung ein Band zwischen Regierung und Bürgerstand erforderlich sei. Damit waren die gegnerischen Anschuldigen nach den Hungerkrawallen klar widerlegt, zugleich hoffte man nun auf ein Entgegenkommen der Regierung während des kommenden Landtags, das dieses Band festigen sollte ${ }^{22}$. Auch wenn es ja kein verbindliches liberales Programm gab, bedeutete diese Weichenstellung angesichts einer zuvor nicht realisierten Bedrohung letztlich einen politischen Kurswechsel, der dann 1848 zu Konsequenzen führen sollte.

19 Albert Eugen Adam, Ein Jahrhundert Württembergischer Verfassung, Stuttgart 1919, S. 80 .

${ }^{20}$ Beob. Nr.160 vom 14.6.1847. Vgl. zu den gegnerischen Angriffen auch Beob. Nr.153 und 159 vom 7.6.1847 bzw. 13.6.1847.

${ }^{21}$ Beob. Nr.184 vom 8.7.1847.

22 Beob. Nr.291 vom 23.10.1847. Vgl. zu den liberalen Überlegungen einer Kanalisierung der Unterschichten, die letztlich jedoch keinen Erfolg versprachen, LANGEWIESCHE (wie Anm. 18) S. 90 und 93. Der Beob. Nr.291 wird dort (S. 90) mit falschem Datum zitiert. 


\section{Ein Rückblick: Die württembergische Opposition bis zur Mitte der 1840er Jahre}

Obwohl Karl Julius Weber 1826 meinte: „In keinem anderen deutschen Staat ist das politische Leben so erwacht wie hier" ${ }^{23}$, war in den Landtagen des Verfassungsstaates Württemberg während der 1820er Jahren von diesem Erwachen wenig zu bemerken - stand doch die 2. Kammer weitgehend „in einem Subordinationsverhältnis“ zur Regierung ${ }^{24}$ und wie diese zudem „unter dem politischen Druck“ des Deutschen Bundes ${ }^{25}$. Bezeichnend war, dass die Beschränkung der Pressefreiheit in der Kammer nicht thematisiert wurde ${ }^{26}$ und diese letztlich - auch im spektakulären Fall des Kammerausschlusses von Friedrich List ${ }^{27}$ - letztlich nur als „Erfüllungsgehilfe“ der Regierung fungierte ${ }^{28}$. Auch Adam konstatiert für jene Zeit „die Willfährigkeit der Stände“; sie hätten „kräftiger auftreten, ihren Wünschen und Beschwerden mehr Ausdruck geben sollen“29. Von einer mehrheitlich „gouvernemental“ orientierten Ständeversammlung ${ }^{30}$ war eine solche Haltung jedoch nicht zu erwarten.

Die Kammerwahl vom Dezember 1831 brachte dennoch der „Bewegungspartei“ einen die Regierung alarmierenden Erfolg: 39 Oppositionellen standen lediglich 32 Regierungsfreunde gegenüber; hinzu kamen die Exponenten des sogenannten „juste milieu“ der Schwankenden, das 22 Köpfe umfasste ${ }^{31}$. Während die Liberalen, vor allem deren führenden Köpfe Duvernoy, Murschel, Pfizer, Schott und Uhland auf eine baldige Landtagseröffnung drängten, setzte die Regierung nicht nur auf eine Verzögerungstaktik, sondern reagierte zusätzlich mit repressiven Maßnahmen, zu denen solche des Deutschen Bundes traten. Zu Letzteren gehörte das Verbot der Gründung von Vereinen, die sich mit politisch-parlamentarischen Fragen beschäftigen wollten. Die Liberalen reagierten am 30. April 1832 mit einer Versammlung in Bad Boll, die von Schott geleitet, das Recht der Stände artikulierte und als „Konvent der Bewegungspartei“ fungierte ${ }^{32}$, obwohl alle gewählten Abgeordneten geladen waren. Die Regierung blieb jedoch unbeeindruckt und raubte so den düpierten Volksvertretern die Möglichkeit, auf das letztlich „europäische

${ }^{23}$ Reise durch das Königreich Württemberg, hier zitiert nach der Neuausgabe Stuttgart 1978, S. 154.

${ }^{24}$ Hartwig Brandt, Parlamentarismus in Württemberg 1819-1870, Anatomie eines deutschen Landtags, Düsseldorf 1987, S. 454.

25 Ebd., S. 460.

26 Ebd., S. 463.

27 Ausführlich dazu ebd., S. 484-495.

28 Ebd., S. 495.

29 AdAm (wie Anm. 19) S. 31.

30 Brandt (wie Anm. 24) S. 500.

31 Diese Richtwerte nach Adam (wie Anm. 19) S. 43.

32 Brandt (wie Anm. 24) S. 507. 
Ereignis“ des Hambacher Festes Ende Mai ${ }^{33}$ angemessen, d.h. im Parlament zu reagieren.

Immerhin bescherte die Boller Versammlung den Liberalen landesweite Aufmerksamkeit; in der Folge wurde eine Art Ausschuss gebildet, der jedoch meist nichtöffentlich wirkte. Brandt spricht hier von der „Konstituierung der liberalen Bewegung als Partei“34.

Während in Württemberg im Sommer eine Welle von Petitionen gegen die Repressionen des Deutschen Bundes einsetzte - die Folgen des Hambacher Festes reagierte König Wilhelm mit der Ernennung Johannes Schlayers zum Innenminister. Er sollte als „starker Mann“ sowohl die Liberalen in Schach halten als auch der Erbitterung im Lande begegnen. In dieser vergifteten Atmosphäre trat am 15. Januar 1833 der vor über einem Jahr gewählte Landtag zusammen, begleitet von hohen Erwartungen einer breiten Öffentlichkeit. Nicht unerwartet verfolgte die Opposition eine Gegenstrategie zum Regierungsplan, zunächst den Staatshaushalt zu behandeln. Mit einer Fülle von z.T. brisanten Motionen, darunter vor allem die von Schott gegen die Pressezensur, von Römer für ein freies Versammlungsrecht und von Pfizer gegen die restriktiven Bundestagsbeschlüsse, löste der erzürnte Monarch am 22. März den so als „vergeblich“ bezeichneten Landtag auf.

Nach den anschließenden, von der Regierung stark beeinflussten Neuwahlen, wurde einigen der Gewählten aus rein politischen Gründen der Urlaub verweigert; Uhland und Römer legten daraufhin ihre Staatsämter nieder. In die am 15. Mai 1833 eröffnete Kammer trat eine leicht geschwächte Opposition und eine besser formierte „Regierungspartei“ ein. Wider Erwarten leiteten bereits die Kommissionswahlen den Prozess der „Entzauberung des Mythos von der liberalen Mehrheit" $\operatorname{ein}^{35}$.

Auf ihren Hauptkampffeldern um die Freiheit der Presse, des Vereins- und Versammlungsrechtes oder gegen das Strafgesetzbuch blieben die Liberalen im „System Schlayer“ weitgehend erfolglos, auch wenn ihre Führerfiguren, vor allem Pfizer, Römer, Schott und Uhland, aber auch Deffner, Duvernoy und Murschel, sich vom „Grauton“ (Hartwig Brandt) des Regierungslagers deutlich absetzten. Der württembergische Liberalismus sah sich so um 1838 ,in einer schweren moralischen Krise“36; seine wichtigsten Repräsentanten resignierten und traten zu den Neuwahlen nicht wieder an. Dieser Rückzug war zweifellos ein Fehler. Noch Jahre später kritisierte der „Beobachter“, er sei keineswegs von günstigen Folgen für das Interesse des Landes gewesen ${ }^{37}$.

33 Vgl. zu dieser größten Massenkundgebung im Vormärz: Veit VALEnTin, Das Hambacher Nationalfest, Frankfurt a. M./Olten/Wien 1982, Zitat S. 65.

34 BrandT (wie Anm. 24) S. 509.

35 Ebd., S. 571.

36 Ebd., S. 602.

37 Beob. Nr. 292 vom 24.10.1847. 
Die Neuwahlen von 1838, erneut von Wahlbeeinflussungen der Regierung begleitet, brachten eine Kammer mit beträchtlicher Mehrheit der „Regierungspartei“, die im Volk als eine Art „Amtsversammlung“ wahrgenommen wurde ${ }^{38}$. Die Opposition war nur mit neun Abgeordneten vertreten, darunter Deffner und Duvernoy, und trat in Kenntnis ihrer Ohnmacht nur noch „gelegentlich“ hervor ${ }^{39}$.

Erst die im November 1844 gewählte neue Kammer, die im Folgejahr erstmals einberufen wurde, markierte eine Regeneration der Opposition. Trotz fortgeführter Wahlbeeinflussungen durch die Regierung waren die Liberalen auf etwa 26 bis 30 Köpfe angewachsen; bei Nachwahlen konnten dann zusätzliche Mandate erzielt werden. Zurückgekehrt waren etwa Römer, der ob seiner Kammertätigkeit bald einen legendären Ruf im Lande errang ${ }^{40}$, ferner Deffner, Duvernoy und Murschel. Neu hinzu kam Friedrich Federer, der als Stuttgarter Kommunalpolitiker und Mitglied der dortigen Bürgergesellschaft ebenfalls zur ersten Garnitur der Liberalen gehörte ${ }^{41}$. Deren Wirken, ihr Umfeld und vor allem ihr Wollen soll nunmehr ausführlich betrachtet werden.

\section{Ein Portrait der liberalen Opposition: Der „Beobachter“, Ziele und Forderungen, Selbstverständnis, Wirkungsfelder}

Ganz ohne Zweifel war der seit 1833 erscheinende „Beobachter“ - ihm war seit 1830 der Hochwächter vorausgegangen - die publizistische Hauptwaffe der Bewegungspartei. Das Volks-Blatt aus Württemberg, wie sein Vorgänger von Rödinger und Tafel gegründet und geleitet, war sozusagen der Popanz der Regierung und ihrer Parteigänger und dementsprechend permanentes Opfer der Zensur, gleichzeitig galten ihm publizistische Anfeindungen durch regierungsfreundliche Blätter $^{42}$. Als Parteiorgan und Oppositionsblatt des Landes par excellence erschien das

38 AdAm (wie Anm. 19) S. 71.

39 BrandT (wie Anm.24) S.609. Ausführlich und mit Hintergründen zur hier nur skizzierten Kammergeschichte seit den 1820er Jahren dort ab S.441. Vgl. auch Adam (wie Anm. 19) S. 17-75 und Walter Grube, Der Stuttgarter Landtag 1457-1957, Stuttgart 1957, S. 509-524.

${ }^{40}$ Vgl. etwa die Verleihung eines sog. Bürgerkranzes als Anerkennung seiner Wachsamkeit um Verfassung und Volksrechte durch seine Anhänger; Beob. Nr.53 vom 23.2.1847. Eine Kurzbiographie des späteren Märzministers bei Frank RABERg (Bearb.), Biographisches Handbuch der württembergischen Landtagsabgeordneten 1815-1933, Stuttgart 2001, S. $734 \mathrm{f}$.

${ }^{41}$ Kurzbiographien der Genannten ebenfalls bei Raberg (wie Anm. 40) S.129f. (Karl Deffner) S.151 ff. (Gustav von Duvernoy), S.194f. (Friedrich Federer), S.596f. (Wilhelm Murschel).

${ }_{42}$ Die Ulmer Schnellpost anerkannte etwa, das Blatt habe sich zwar durch seine rücksichtslose Opposition auf die Mißbräuche im Lande Verdienste erworben, fand jedoch Art und Form der Kritik tadelnswert; Beob. Nr.21 vom 23.1.1846. 
Stuttgarter Blatt täglich und wurde landesweit gelesen; Korrespondenten - zumeist Parteigänger - berichteten aus ganz Württemberg, um dessen engen faulen $\mathrm{Zu-}$ ständen wenigstens das Salz hinzuzufügen ${ }^{43}$. Dabei galt der Grundsatz, dass Thatsachen und Persönlichkeiten als Fleisch und Blut der politischen Diskussion empfunden wurden, sei doch andernfalls der Gott des Schlafes der Gebieter ${ }^{44}$. Die Entschiedenheit der politischen Meinung gelte der Sache unseres Volkes - und dafür habe man seit den Anfängen schon Außerordentliches geleistet ${ }^{45}$.

In religiöser Hinsicht forderte der „Beobachter“ entschieden das Ende staatlicher Bevormundung der Kirchen ${ }^{46}$. Gleichzeitig polemisierte er häufig und zornerfüllt gegen die Umtriebe der württembergischen Pietisten. Deren antiemanzipatorisches Wirken als widernatürliche Richtung christlichen Lebens mache es zur Pflicht, diesem Verfinsterungssysteme entschieden entgegenzutreten ${ }^{47}$.

Der seit 1843 als Redakteur des „Beobachters“ wirkende Adolph Weisser ${ }^{48}$ sah sich schon kurz nach seinem Dienstantritt im Vorfeld der Landtagswahl wüsten „Beschimpfungen“ ausgesetzt ${ }^{49}$. Nach den bereits erwähnten Hungerkrawallen von 1847 begann ein gegnerisches Trommelfeuer gegen das Blatt und die Liberalen, die als Drahtzieher der Ausschreitungen bezichtigt wurden. Bei der Abwehr stand Weisser in vorderster Linie; er gehörte fraglos zu den Schlüsselfiguren der Opposition $^{50}$.

Gegen die vielfachen Vorwürfe der gegnerischen Presse, das Blatt verhalte sich destruktiv, da es immer nur verneine und so den Staat gefährde, setzte Weisser sein und seiner Partei Credo des eigenen Wollens, das bereits ins Blut des Volkes übergegangen sei. Man erstrebe nicht das verderbliche Halbe, sondern das Ganze. Dazu müsse zuvor das Alte weggeräumt werden, um das Neue einführen zu können. Zensurbedingt reagiere das Blatt mitunter verbissen, dennoch gelte, dass je mebr Belebrung und Licht sich verbreitet, desto mehr die Blindheit alles Autoritäts-Glaubens verschwinden wird ${ }^{51}$.

${ }^{43}$ Beob. Nr. 172 vom 27.6.1846.

${ }^{44} \mathrm{Ebd}$.

45 Beob. Nr.21 vom 23.1.1846.

${ }^{46}$ Beob. Nr. 19 vom 21.1.1846.

47 Beob. Nr. 149 vom 4. 6. 1846. Vgl. auch die Nummern 137, 189 und 190 vom 21.5.1846, 13.7.1846 und 14.7.1846. - Da die Pietisten politisch letztlich im Regierungslager standen (vgl. dazu etwa Raimund Waibel, Frühliberalismus und Gemeindewahlen in Württemberg 1817-1855, Stuttgart 1992, S.346f.) waren sie für die Liberalen sozusagen Reaktionäre im Doppelpack.

48 Beob. Nr.21 vom 23.2.1846.

49 Vgl. dazu Waibel (wie Anm. 47) S. 340.

50 Waibel zählt einen seiner entsprechenden Artikel „zu den Glanzlichtern des württembergischen Journalismus im Vormärz“; ebd., S.366f., Anm. 863.

${ }^{51}$ Beob. Nr. 185 vom 9.7.1847. 
Angesichts inzwischen verschärfter Zensur ${ }^{52}$ und des erscbwerte[n] Recht [s] der öfentlichen Versammlung beneidete man Baden wegen der dort vorherrschenden besseren Verhältnisse ${ }^{53}$; sei doch in Württemberg die oppositionelle Presse Dank der Censur eine abgestumpfte Waffe und tue Niemand webe ${ }^{54}$. Allerdings wurde dieser Befund relativiert: Die ungemeine Verbreitung des Blattes im Lande sorge dafür, das jedwede Rohbeit, Willkürakte oder Rechtsbeugungen und Unterdrückung jeweils zu Berichten der Korrespondenten führe ${ }^{55}$. Dies bedeutete in der Tat, dass das Oppositionsblatt seine selbstgestellte Aufgabe, ein Wächteramt zu bekleiden, tatsächlich erfüllte.

Es versteht sich so, dass der „Beobachter“ für die Liberalen ein unverzichtbares Gegengewicht zur größten Zeitung des Landes, dem „Merkur“, darstellte. Dieser war zwar konstitutionell, aber letztlich doch regierungsfreundlich ${ }^{56}$. Während die zumeist auf Bezirksebene erscheinenden sogenannten Intelligenzblätter sich z.T. bereits seit den 1830 er Jahren mit Fragen lokaler Politik beschäftigten ${ }^{57}$, blieb dort die Landespolitik einschließlich der Wahlen „streng tabu“ ${ }^{\text {58 }}$. Dennoch trugen auch die Intelligenzblätter im Rahmen ihrer eingeschränkten Möglichkeiten zur Verbreitung liberalen Wollens bei. Insgesamt unterstreicht das oben Gesagte die ganz und gar exzeptionelle Rolle des „Beobachters“ im Vormärz - er war zur „Institution geworden“ 59 , die auch einem Gottlieb Rau ihre Spalten öffnete.

Die liberale Opposition in Württemberg präsentierte sich am Vorabend der Revolution keineswegs schlagkräftig und homogen. Da organisierte Parteien im heutigen Sinne noch nicht existierten - auch wenn der Begriff in zeitgenössischen Texten häufig auftaucht ${ }^{60}$, ist realiter von einer "Gesinnungsgemeinschaft“ zu sprechen $^{61}$. Zugleich galt, dass die Liberalen im Königreich etwa im Vergleich zu

52 Beob. Nr.291 vom 23.10.1847.

53 Beob. Nr.292 vom 24.10.1847.

${ }^{54}$ Beob. Nr.331 vom 3.12.1847.

55 Beob. Nr.293 vom 25.10.1847.

56 Vgl. etwa Brandt (wie Anm. 24) S. 108.

57 Vgl. Werner StröвELe, Die Anfänge eines Mediums, Zur Geschichte des lokalen Teils in den württembergischen Zeitungen des 19. Jahrhunderts und dessen Fallbeispiel der Tübinger Chronik, in: Hans-Peter BIEge (Hg.), Massenmedien in Baden-Württemberg, Stuttgart 1990, hier S. 73 und S. 80-86, ferner Philippe Alexandre, Schwäbisch Hall im Vormärz (1830-1848), Gesehen durch seine Zeitungen, Schwäbisch Hall 1993.

${ }^{58}$ Strö̈ele (wie Anm. 57) S. 80. Dort zur Zensur S.77-80. Vgl. zur Zensur auch Gregor Richter, Der Staat und die Presse in Württemberg bis zur Mitte des 19. Jahrhunderts, in: ZWLG 25 (1966) S.394-425.

59 BRAndt (wie Anm. 24) S. 136.

60 Der „Beobachter" definierte etwa, die Parthei aber ist nichts Anderes als eine lebendig gegliederte Organisation gemeinsamer Bestrebungen; Nr.228 vom 21.8.1847.

${ }_{61}$ Dazu Langewiesche (wie Anm. 18) S. 82 (Zitat) f. 
Baden nicht nur unzureichend organisiert waren ${ }^{62}$, sondern im „Beobachter“ auch eine Zersplitterung und zwei Schattirungen konstatiert wurden ${ }^{63}$.

Hier wird vage ein Zustand angedeutet, den Stadelmann prägnant beschrieben hat: Wie im übrigen Deutschland war auch in Württemberg die Opposition „bis 1848 noch ungeschieden vereinigt“, während in „politisch fortgeschrittenen“ Ländern Europas bereits „parteimäßig und weltanschaulich“ eine Differenzierung stattgefunden hatte. Für den Deutschen Bund dagegen galt, dass hier noch „demokratische und liberale Tendenzen, republikanische und ständische Motive, romantische und aufklärerische Vorstellungen, freihändlerische und zünftlerische Argumente durcheinander wogten “64.

Einigkeit herrschte im liberalen Lager dagegen beim Grundziel der „Überwindung des Absolutismus und der feudalen Gesellschaftsstruktur“ 65 oder wie es Murschel formulierte darin, die Verbeißungen der Verfassung geltend zu machen. Dazu gehörte nicht nur für ihn auch eine soziale Komponente; er habe sich stets für eine Erleichterung der Steuern und Abgaben und die Befreiung von Grund und Boden eingesetzt ${ }^{66}$.

Die Erwartungen der Liberalen zielten am Vorabend der Revolution auf eine umfassende Reform des gesamten Staatswesens; der „Beobachter“ hatte dies Anfang 1847 schnörkellos artikuliert: Obne die Erneuerung und Reorganisation des socialen, kirchlichen und sittlichreligiösen Lebens [könne] auch das politische Leben auf keine nachbaltige und gründliche Heilung boffen ${ }^{67}$. Im Herbst des Jahres erklärte Römer während einer Advokaten-Versammlung in Gmünd unwidersprochen, sein Berufsstand stehe in Gegnerschaft zum abstrakten Beamtenstaat; er forderte seine Kollegen auf, am allgemeinen Streben nach der Reform des ganzen Staates in freieren Sinne teilzunehmen ${ }^{68}$.

Das Gros ihrer Ziele und Forderungen - eben jene „Verheißungen der Verfassung“ in Wort und Geist - galten den Liberalen als längst ins Volksbewusstseyn eingedrungene Lebensfragen ${ }^{69}$. Zentral war die geforderte Abschaffung der Wahlbeeinflussungen durch die Regierung, die Beseitigung der Feudallasten, die öffentliche und mündliche Verhandlung bürgerlicher Rechtsstreitigkeiten und die Einführung von Schwurgerichten, schließlich die seit langem reklamierte Freiheit der

\footnotetext{
62 Vgl. Beob. Nr.292 und 293 vom 24.10.1847 und 25.10.1847.

63 Beob. Nr.322 vom 23.11.1846.

64 Stadelmann (wie Anm.2) S.31. Als Beispiel dafür können die unterschiedlichen Ansichten der württembergischen Liberalen zum Zollvereinsbeitritt 1833 gelten. Vgl. dazu Brandt (wie Anm.24) S.604.

65 Stadelmann (wie Anm. 2) S. 31.

66 Beob. Nr.305 vom 6.11.1846.

67 Beob. Nr. 25 vom 26.1.1847.

68 Beob. Nr. 288 vom 20.10.1847.

69 Beob. Nr. 1 vom 1.1.1846. Hier werden zahlreiche Forderungen, die ansonsten immer wieder erhoben wurden, artikuliert.
} 
Presse sowie des Vereins- und Versammlungsrechtes. Auf kommunalem Gebiet erstrebte man sowohl die Abschaffung lebenslänglicher Gemeinderäte als auch die Öffentlichkeit der gemeinderätlichen Verhandlungen ${ }^{70}$. Dieser größtenteils seit Jahren erfolglos artikulierte Forderungskatalog, in Einzelfällen bestenfalls mit unbefriedigenden „Abschlagszahlungen“ bedacht, fand so Eingang in die vielzitierte, von Uhland verfasste Adresse der Tübinger Bürgerschaft an den ständischen Ausschuss vom 2. März $1848^{71}$.

Der im Oktober 1846 verstorbene Esslinger Fabrikant Karl Deffner verkörperte für die Liberalen - und wohl über sie hinaus - das Idealbild eines Abgeordneten. Er sei seinen Arbeitern ein milder und väterlicher Herr gewesen, habe in der Kammer und auch außerhalb vermittelnd und uneigennützig gewirkt und so im ganzen Land eine schmerzliche Lücke hinterlassen. Seiner Beerdigung wohnten nicht nur Vertreter der Regierung, sondern auch Trauergäste aller politischen Richtungen bei; er wurde trotz seines großen Einflusses als schlichter Bürger und Mann des Volkes gerühmt ${ }^{72}$.

Wilhelm Murschel beschrieb dagegen sein Selbstverständnis als Abgeordneter mit anderen Akzenten. Der neben Römer als die größte agitatorische Begabung in der Partei und einer ihrer Populisten Charakterisierte ${ }^{73}$ betonte, er wirke in dem Bewusstsein, nicht von der Regierung, sondern vom Volke entsandt zu sein, um allein für das Wobl des Landes und des Volkes, also im Dienste des Rechtes und der Freibeit tätig zu sein. Dabei sei die gegnerische Unterstellung, die Opposition würde allen Regierungsinitiativen ablehnend begegnen, völlig unzutreffend. Diese habe vielmehr auf das Bereitwilligste solchen Anträgen der Regierung zugestimmt, die sie für gut befinden konnte ${ }^{74}$.

Generell sahen sich die Liberalen nicht nur bei Wahlanfechtungen im Vergleich zu den Regierungsfreunden schlechter behandelt ${ }^{75}$, die Elemente und Gliederungen der Staatsgewalt repräsentierten zudem auch außerhalb der Kammer letztlich die konservative Partei ${ }^{76}$. Grundsätzlich gehörte für die Opposition die Notbwen-

${ }^{70}$ Vgl. zum liberalen Prinzip der Öffentlichkeit in der gesamten Staatsverwaltung Waibel (wie Anm. 47) S.118ff.

71 Abdruck bei Hermann Bausinger (Hg.), Ludwig Uhland, Ausgewählte Werke, München 1987, S.328-330. Auf die dort ebenfalls artikulierten nationalpolitischen Ziele wird noch eingegangen.

72 Beob. Nr.296 vom 28.10.1846 und Nr. 298 vom 30.10.1846.

73 Brandt (wie Anm. 24) S. 592.

74 Beob. Nr.305 vom 6.11.1846 (Wahlrede in Plochingen), vgl. auch Beob. Nr.324 vom 25.11.1846.

75 Römer im Landtag, Beob. Nr.15 vom 16.1.1847, vgl. auch Beob. Nr.108 vom 20.4.1847.

76 Beob. Nr. 246 vom 8. 9.1847. In diesem Kontext stellte sich aus liberaler Sicht auch die Frage der Wünschbarkeit von Staatsdienern als Abgeordnete. Man sei nicht blindlings dagegen, wäre dies doch eine Versündigung an der Intelligenz; Beob. Nr.340 vom 12.12.1846. Allerdings ergebe sich ein Dilemma: Staatsdienern, die die Liberalen unterstützten, drohe 
digkeit des Kampfes zum Prinzip des Verfassungsstaates. Die neue konservative Doktrin vom innigsten Einverständnis zwischen Regierenden und Regierten habe allein zum Ziel, den constitutionellen Sinn des Volkes einzuschläfern ${ }^{77}$.

Die Liberalen hatten schon früh die Kommunalpolitik als wichtiges Betätigungsfeld besetzt. Seit den 1830 er Jahren konnten sie nicht nur in Stuttgart das regelmäßig zu erneuernde kommunale Kontrollgremium, den Bürgerausschuss, sozusagen als eigenes „Organ“ gegenüber den mit „Lebenslänglichen“ besetzten Gemeinderäten etablieren ${ }^{78}$. Im Gemeindeleben sah der „Beobachter“ eine staatsbürgerliche Schule und den Quell, wo alles politische Leben warm und in seinen bedeutungsvollsten Anfängen hervorsprudelt ${ }^{79}$.

Der liberale Einsatz gegen die „Lebenslänglichen“ und für öffentliche Verhandlungen waren z.T. über das liberale Milieu hinaus im Land überaus populär. In einer Petition aus Murrhardt an die Kammer wurden die beiden Desiderate als Zeitbedürfnisse bezeichnet, die sowohl die Gemeinden als auch das ganze Volk beträfen ${ }^{80}$. Zwar konstatierte das Parteiorgan im Mai 1846 reißende Fortschritte im Gemeindeleben $^{81}$, die beiden kommunalen Zentralforderungen scheiterten jedoch trotz Kampagnen und Landtagsinitiativen bis 1848 an der Intransigenz der Regie$\operatorname{rung}^{82}$.

Vor dem Hintergrund des Verbots politischer Vereinigungen und Versammlungen seit $1832^{83}$ sang der „Beobachter“ ein Hohelied auf die bestehenden Bürgervereine in Stuttgart, Esslingen, Gmünd und Waiblingen und plädierte für weitere Gründungen. Vom Stuttgarter Verein gehe der Impuls fast zu allen Regungen des politischen Lebens aus; er sei ein Zentrum öffentlichen Lebens ${ }^{84}$. Zwar betonte das Blatt, die Bürgergesellschaft als solche betreibe keine Politik, gehörten doch die Mitglieder zu allen politischen Farben. Dennoch sei dort das liberale Prinzip das vorherrschende, auch wenn es nur auf der Privatthätigkeit Einzelner fuße $\mathrm{e}^{85}$. Waibel sieht in der Stuttgarter Bürgergesellschaft den „organisatorische[n] Rahmen“ der Opposition und verweist darauf, dass u.a. Wilhelm Murschel, ein verdienter

\footnotetext{
wie etwa Römer oder Uhland die Urlaubsverweigerung. Wo dies nicht der Fall sei ständen die Beamten in der Regel im Regierungslager. Daher sei man im Allgemeinen (im Original gesperrt) gegen solche Nominierungen; Beob. Nr. 263 vom 25. 9.1847.

77 Beob. Nr. 250 vom 12.9.1847.

78 Waibel (wie Anm. 47) S.304f.

79 Beob. Nr. 138 vom 23.5.1846.

80 Beob. Nr. 35 vom 5.2.1847.

${ }^{81}$ Beob. Nr. 138 vom 23.5.1846.

${ }^{82} \mathrm{Zu}$ beiden Komplexen ausführlich WaibeL (wie Anm. 47) S.79-102 zur „Lebenslänglichkeit", zur Öffentlichkeit S.118-126.

83 Dazu Brandt (wie Anm. 24) S. 304.

${ }^{84}$ Beob. Nr.60 vom 2.3.1847.

85 Beob. Nr.63 vom 5.3.1847.
} 
Matador (nicht nur) der Stuttgarter Kommunalpolitik ${ }^{86}$, dort zeitweise als Vorsitzender fungierte ${ }^{87}$.

In solch „kryptoparteilichen“ Vereinen, in landesweit verbreiteten Schützenvereinen, Lese- und Bürgergesellschaften sowie Gewerbevereinen sind liberale Einflüsse durch das dort überwiegend vertretene „höhere“ Bürgertum nachweisbar ${ }^{88}$. In Hall trug etwa der Jurist Eduard Schübler ${ }^{89}$, bekennender und praktizierender Liberaler, maßgeblich als Impulsgeber zur „Politisierung“ des dortigen Bürgertums bei. Er war 1819 und 1844 bis 1848 Kammermitglied, Publizist und Kommunalpolitiker sowie Gründer des Haller Gewerbevereins, in dessen Bibliothek auch der „Beobachter“ auslag90.

Die württembergischen Liberalen knüpften schon frühzeitig Kontakte zu ihren Gesinnungsgenossen über die Landesgrenzen hinaus. So trafen sich im Juni 1833 Pfizer, Römer, Schott und Uhland mit Parteifreunden aus Baden und HessenDarmstadt im badischen Langenbrücken, um über den Zollverein und dessen politische Auswirkungen zu diskutieren; Schott prognostizierte im Anschluss an das Treffen „einen harten Kampf“ in der württembergischen Kammer"

Auf dem Weingut des badischen Abgeordneten von Itzstein in Hallgarten im Rheingau trafen sich seit 1839 südwest- und norddeutsche Parlamentarier darunter auch Württemberger - konspirativ zum Meinungsaustausch etwa über ihre Taktik in den Landtagen. Der sich erweiternde Kreis wurde schließlich von Politikern demokratischer Couleur dominiert ${ }^{92}$.

Besser unterrichtet sind wir über das Treffen liberaler Parlamentarier aus verschiedenen deutschen Staaten am 10. Oktober 1847 im hessischen Heppenheim, an dem aus Württemberg Federer, Fetzer, Goppelt, Murschel und Römer teilnahmen.

86 Vgl. dazu etwa Beob. Nr. 3 vom 4.1.1847.

87 Waibel (wie Anm.47) S.236 mit Anm. 90. Dort ausführlich zur Bürgergesellschaft S.232ff. Zur Esslinger Bürgergesellschaft, durch ihren Mitbegründer Deffner „freisinnig ausgerichtet" siehe Heinrich Tiessen, Industrielle Entwicklung, gesellschaftlicher Wandel und politische Bewegung in einer württembergischen Fabrikstadt des 19. Jahrhunderts. Esslingen 1848-1914 (Esslinger Studien, Bd.6), Esslingen 1982, S. 90.

88 Waibel (wie Anm. 47) S.14f.

89 Zu ihm Philippe Alexandre, Eduard Schübler (1792-1870), Jurist, Politiker und Theoretiker des „organischen Staates“. Ein Beitrag zur Geschichte des Frühliberalismus in Württemberg, in: Württembergisch Franken, Bd. 90/91, Schwäbisch Hall 2007, S.327-360.

90 Alexandre, Schwäbisch Hall im Vormärz (wie Anm. 57) S. 105. - Schübler war auch Exponent der landesweiten Polenbegeisterung von 1831/1832, einer durchaus liberal geprägten Manifestation, bei der er sich durch Mithilfe bei der Sammlung von Geld und Sachspenden für die durchreisenden unglücklichen Revolutionäre engagierte; ebd., S.31.

${ }^{91}$ Dazu Hans-Werner HaHN, Zwischen deutscher Handelsfreiheit und Sicherung landständischer Rechte. Der Liberalismus und die Gründung des Deutschen Zollvereins, in: Wolfgang Schieder (Hg.), Liberalismus in der Gesellschaft des deutschen Vormärz, Göttingen 1983, S.239-271, hier S.246f. Vgl. auch Anm. 64.

92 Dazu Siegfried Schmidt, Hallgarten-Kreis (HK) 1839-1846, in: Lexikon zur Parteiengeschichte, Bd.3, Leipzig 1885, S. 81-83. 
Zweck der Gespräche war einerseits eine Koordinierung bezüglich der deutschen Nationalangelegenbeiten, andererseits zur Abbülfe der gegenwärtigen allgemeinen Uebelstände Strategien für die kommenden Landtagsverhandlungen zu entwickeln. In der deutschen Frage seien zwar eine Nationalvertretung und eine Nationalregierung wünschenswert, weitaus realistischer erschien es jedoch, hier zunächst eine Ausbildung des Zollvereins zu einem deutschen Vereine anzustreben. Diesen gelte es dann fortzuentwickeln und so einen Keim zu schaffen, der die Möglichkeit biete, eine wabre deutsche Macht [zu] begründen.

Bezüglich der in den einzelstaatlichen Kammern zu verfolgende Strategie ergab sich eine erfreuliche Uebereinstimmung. Dort sollten möglichst gleichlautend die seit Jahren erhobenen Forderungen - von der Pressefreiheit bis zur Abschaffung der Feudallasten sowie eine Minderung der Militärausgaben - artikuliert werden. Eine Kommission, der für Württemberg Federer angehörte, sollte für eine größere Versammlung im kommenden Jahr Vorbereitungen treffen. Dort würden auch die Zustände der ärmeren Klassen, Erleichterungen für den kleineren Mittelstand und für die Arbeiter zur Sprache kommen ${ }^{93}$. Gegnerische Kritik der liberalen Götzendiener wurde zurückgewiesen. Das Oppositionsblatt betonte, selbst die entschiedenste ständische Opposition in Deutschland hat sich gewöhnt, unter allen Umständen einen streng loyalen Gang einhalten. So sei die Versammlung lediglich das bescheidene Lebenszeichen deutscher Einigkeit gewesen ${ }^{94}$. Dies hieß im Klartext, dass in Heppenheim ein Reform- und keineswegs ein Revolutionsprogramm verabredet wurde.

\section{Regierung und Opposition im Zeichen des drohenden "Gewitterhimmels“}

Bilanziert man - vor dem Hintergrund der tiefen sozio-ökonomischen Krise den politischen Zustand Württembergs seit etwa der Mitte des Jahres 1847, so wird überdeutlich, dass weder Regierung noch Opposition gewappnet waren, der erkennbaren dumpfe[n] Schwere eines Gewitterbimmels, die der „Beobachter“95 ahnungsvoll konstatiert hatte, wirkungsvoll begegnen zu können.

So zeichneten die im Parteiblatt abgedruckten Württembergischen Briefe der liberalen Deutschen Zeitung angesichts der materiellen Not ein düsteres Zukunftsbild und bestätigten so die Warnrufe von Gotttlieb Rau. Hingewiesen wurde auf die z.T. dramatische Zunahme der Konkurse, die Noth der arbeitenden Klassen sowie die handelspolitische Entwicklung, die einen großen Teil der Bevölkerung in einen Helotenzustand versetze. Daraus resultiere Haß gegen die Reichen und

\footnotetext{
93 Beob. Nr. 286 vom 18.10.1847.

94 Beob. Nr.302 vom 3.11.1847.

95 Beob. Nr. 1 vom 1.1.1848.
} 
Mißtrauen gegen die bestebende Gewalt ${ }^{96}$. Die Regierung sehe sich mit in's Unmögliche gesteigerten Ansprüchen konfrontiert, obwohl sie etwa an den Missernten keine Schuld trage, jedoch durch ihre überbordende Bürokratie den Unmut schüre ${ }^{97}$. Letztlich könne nur sie den peinlichen Zustand des Landes beenden ${ }^{98}$.

Eine vehemente Kritik am württembergischen Staatsleben formulierte ein anonymer Autor, dem der „Beobachter“ über mehrere - zensierte - Nummern seine Spalten öffnete ${ }^{99}$. Er sah die Wurzel aller Uebel in der die besten Staatskräfte verzehrenden Centralisation, dem Hinaufziehen zu vieler Geschäfte von den untergeordneten Lokalstellen zu den böheren Centralbehörden. Daher forderte er als Heilmittel vor allem die gemeindliche Selbstverwaltung, verbunden mit regelmäßiger Wahl der Ortsvorsteher und Gemeinderäte, deren Kompetenzen zu erweitern seien. Bisher fehle den bevormundeten kommunalen Verwaltungen sowohl das Vertrauen des Volkes als auch der Regierung. Beklagt wurde ferner der traurige Zustand der Rechtspflege, während der mit Kleinkram belasteten Innenverwaltung Zeit für die großen Dinge fehle. Auch im Finanzwesen befinde sich durch die Zentralisierung vieles im Argen; dort werde das Große wie eine Kleinigkeit behandelt. Wirklich wichtig sei dagegen die Schaffung einer Kreditkasse für die Ablösung der Feudallasten sowie einer Nationalbank für die Gewerbe. Dieses Plädoyer für Dezentralisierung und Straffung ging letztlich dahin, der reformunwilligen württembergischen Staatsverwaltung eine wirkliche Zukunftsfähigkeit abzusprechen. Diese und das Vertrauen des Volkes seien nur zu erzielen, wenn man zuvor den Schutt und das Baufällige entferne.

Die württembergische Opposition befand sich nach den erwähnten Hungerkrawallen vom Frühjahr 1847 zunächst in einen Zustand der Ratlosigkeit und Furcht ${ }^{100}$, sah man sich doch völlig unvorbereitet, dem drohenden Sturme zu begegnen $^{101}$. Von außen wurde ihr bescheinigt, dass nicht nur die Geister stark auseinander gingen, sondern es auch an politischer Thatkraft fehle ${ }^{102}$. Im Oktober 1847 setzte man angesichts der communistischen Gährung Hoffnung darauf, dass im kommenden Landtag das Band zwischen Regierung und Bürgerstand befestigt werde ${ }^{103}$. Einige Wochen später wurde dann die liberale Neuorientierung präzise formuliert: Die Verschmelzung der Interessen von Bürgertum und Regierung sei die beste und sicherste Schutzwehr gegen das Andringen des Proletariates, das nur

96 Beob. Nr. 284 vom 16.10.1847.

97 Beob. Nr.285 vom 17.10.1847.

98 Beob. Nr.286 vom 18.10.1847.

99 Das Folgende aus den Nummern 355 bis 358 vom 28.12. bis 31.12.1847 (Was will das Volk in Württemberg?).

100 Vgl. dazu etwa Beob. Nr. 136 vom 19.5.1847 (Beilage), Nr. 153 vom 7.6.1847, Nr. 159

vom 13.6.1847 und Nr. 160 vom 14.6.1847.

101 Beob. Nr.184 vom 8.7.1847.

102 Beob. Nr. 286 vom 18.10.1847.

103 Beob. Nr. 291 vom 23.10.1847. 
durch einen solchen Wall in die ihm gebübrenden Grenzen zurückgedrängt werden könne. Stehe doch fest, dass jedwedes Zugeständnis nur zu nenen Forderungen führen werde ${ }^{104}$.

Der liberale Kurswechsel, verbunden mit der Hoffnung auf ein Entgegenkommen der Regierung und zugleich ein Versuch, „den drohenden Verfall der bürgerlichen Einheitsfront zu verhindern" ${ }^{105}$, wurde dann 1848 zu einem Mosaik bei der hier nicht mehr zu behandelnden Spaltung der Opposition, der Trennung von Liberalen und Demokraten ${ }^{106}$.

Gravierend war dieser Kurswechsel insofern, als er die Abkehr der (süddeutschen) Liberalen von ihrem früheren, allerdings längst brüchig gewordenen Konzept „einer klassenlosen Bürgergesellschaft ,mittlerer’ Existenzen“ markierte und nun, angesichts veränderter sozialer und wirtschaftlicher Herausforderungen, den Weg zur „Klassenpartei“ eröffnete ${ }^{107}$.

Das Parteiblatt - und was die Leitartikel betrifft, war dies Redakteur Weisser reagierte auf konstruktive Kritik durchaus zugänglich. Feststellungen wie etwa die zu seltene Behandlung liberaler Reformvorhaben, der Partei fehle eine durchgreifende Organisation, ihrem Organ eine systematische Unterstützung, zentrale Artikel oblägen ausschließlich dem Redakteur während sonstige Beiträge häufig Zufallsprodukte seien, enthielten, so Weisser, viel Wabrheit. Er gab jedoch zu bedenken, dass der alltägliche Kampf mit der Zensur zermürbe und fast zum Wunsch führe, die Feder zur Seite zu legen ${ }^{108}$.

Anfang Dezember 1847 beschäftigte sich das Blatt über drei - zensierte - Ausgaben ausführlich mit der Stellung der Opposition - die Antwort auf Kritik aus unterschiedlichen Lagern. Ihr solle weder ein Dornenkreuz noch eine Lorbeerkrone gewunden werden, vielmehr sei zu erläutern, warum eine wünschenswerte effizientere Haltung kaum möglich sei. Letztlich, so lautete das Fazit, verhinderten der württembergische Volkscharakter, das herrschende System und die derzeitige Not größere Erfolge ${ }^{109}$.

104 Beob. Nr.317 vom 18.11.1847. - Ausführlich dazu Langewiesche (wie Anm.18) S. 86-90, der auch auf liberale „Überlegungen“ verweist, das Proletariat wieder unter die Fittiche des Bürgertums zu bringen; ebd., S. 90.

105 Langewiesche (wie Anm. 18) S. 90.

106 Dazu etwa Brandt (wie Anm. 24) S.615f., Hans Peter Müller, Carl Mayer (18191889) - ein württembergischer Gegner Bismarcks. 1848er, Exilant, demokratischer Parteiführer und Parlamentarier, Stuttgart 2014, S.11 f. und ausführlich LANGEwiEsche (wie Anm. 18) S. 151-220.

107 Lothar GaLl, Liberalismus und „bürgerliche Gesellschaft“. Zu Charakter und Entwicklung der liberalen Bewegung in Deutschland, in: DERs. (Hg.), Liberalismus, Köln 1976, S. 162-86, hier S. 176.

108 Beob. Nr. 293 vom 25.10.1847.

109 Beob. Nr.333 vom 5.12.1847. 
Böswillige Vorwürfe wie etwa Meinungsterror und Phrasendrescherei wurden angesichts der übermächtigen Zensur als Perfidie zurückgewiesen; sie wären ohnehin nur einer woblorganisirten und systematischen Opposition möglich. Einem wohlgesonnenen Kritiker wurde bedeutet, die von ihm vermissten politischen Hebel seien in der Realität nicht vorhanden. Daher sei der Boden zu untersuchen, auf dem die Liberalen wurzelten ${ }^{110}$.

Dies führte zu einer Betrachtung des Volkscharakters, die fast einer volkskundlichen Studie glich. Konstatiert wurde ein egoistischer Grundzug - man sei immer zu nebmen bereit, selten zu geben. Das Vereinsleben sei nirgends geringer als im Lande, im öffentlichen Leben sei der Württemberger unpatriotisch, Gemeinde und Staat erschienen ihm als Anstalten, die eine Gegenleistung für bezahlte Abgaben schuldeten. Man sei nicht feig, aber sorglich und befürchte, Schaden zu erleiden. Fäuste im Sack seien allgegenwärtig; der Liberalismus säße im Herzen, nicht im Kopf, liberales Handeln falle so schwer und allzu viele betrachteten Wahlen als eine Art politischen Zeitvertreib. Da die Freibeit nur in langem Guerillaskrieg zu erobern sei, scheue man die Beschwerden eines solchen Kampfs ${ }^{111}$.

Während das System der polizeilichen Wohlfahrtsbeförderung bereits die Entwicklung eines wirklich konstitutionellen Lebens behindere, stelle die Not im Lande eine Herausforderung dar. In dieser Situation fehle der Mehrheit der Sinn für die Fragen der bloßen Politik. Vertrauten die einen der Regierung, glaubten andere nicht mehr an eine friedliche Lösung, sondern wandten sich zum Proletariat und sahen ibr Heil im Umsturz. In den politischen Reformen des Liberalismus wollten nur Wenige die Rettung erkennen.

Schließlich verteidigte das Blatt das Verbalten der Opposition als ehrenhaft, gestand jedoch zu, dass ihr Operieren durchaus verbesserungswürdig sei. Allerdings gelte es zu bedenken, dass unter den bestehenden Verhältnissen eine effizientere Parteiorganisation fast zur Unmöglichkeit werde. Dem Liberalismus eine schärfere Spitze zu geben berge zudem die Gefahr einer Spaltung seiner Anhänger und gebe den gegnerischen Terrorismusvorwürfen neue Nahrung ${ }^{112}$.

In der Tat war hier ein Dilemma formuliert worden, das deutlich machte, dass der württembergische Liberalismus sich am Vorabend der Revolution keineswegs schlagkräftig präsentierte. Auch die Tatsache, dass im Januar 1848, vor der Eröffnung des Landtages, vielerorts Wählerversammlungen den Katalog liberaler Forderungen guthießen ${ }^{113}$, konnte letztlich die Schwäche der Opposition nicht kaschieren. Sie war, ebenso wie die Regierung, für die vom „Beobachter“ konstatierte Gewitterstimmung nicht gerüstet - aus den oben angeführten Gründen konnte sie es nicht sein.

110 Beob. Nr.331 vom 3.12.1847.

111 Beob. Nr.332 vom 4.12.1847 (z.T. gesperrt).

112 Beob. Nr.333 vom 5.12.1847.

113 Vgl. zu Stuttgart etwa Beob. Nr.19 vom 21.1.1848. 


\section{Abschließende Betrachtungen}

Als sich im März 1848 jener Gewitterhimmel als „Sturm, der in die Zeit gefahren ist“ - so Uhland - präsentierte, schien sich ein großer Sieg der Opposition anzubahnen. War doch für König Wilhelm die erzwungene Berufung des „Märzministeriums“, mehrheitlich aus den zuvor bekämpften Liberalen - Römer, Duvernoy, Goppelt und Pfizer bestehend - nicht nur ein demütigender $\mathrm{Akt}^{114}$, sondern auch die Quittung für die seitherige Intransigenz und Reformunwilligkeit, ja letztlich eine politische Bankrott-Erklärung. Die neuen Minister ${ }^{115}$, alle Exponenten eines antirevolutionären Reformkurses, verkörperten nun das erstrebte „Band“ mit der Staatsführung und konnten sich als Vertreter des ebenfalls auf Reformen setzenden liberalen Establishments fühlen. Die unausweichliche Trennung der Demokraten von diesem Kurs des Establishments sprengte und schwächte die bürgerliche Einheitsfront erheblich.

Mit ihren unterschiedlichen Strategien - die Liberalen setzten auf „Vereinbarung “ und waren dabei kompromissbereit, die Demokraten steuerten einen mehr kleinbürgerlich orientierten, aber zunächst ebenfalls antirevolutionären Kurs der „Konfrontation“ - scheiterten schließlich beide Seiten ${ }^{116}$.

Beschränkt man den Blick auf Württemberg, muss man beiden Richtungen Naivität bescheinigen. Ein Zeitgenosse bemerkte für die Frühphase der Revolution lakonisch: „An die Hauptsache, die Machtfrage, dachte fast niemand“117. Die königlichen Zugeständnisse vom März 1848 wurden - auch von den Demokraten allzu lange - als ehrlicher Ausdruck der Reformwilligkeit interpretiert, während sie in Wirklichkeit situationsbedingt letztlich nur taktischer Art waren. Spätestens mit der Sprengung des „Rumpfparlaments“ im Juni 1849 war deutlich geworden, dass die Regierung - bis Ende Oktober noch von Römer geführt! - im Windschatten der gesamtpolitischen Entwicklung wieder auf reaktionärem Wege war, der dann spätestens mit der Berufung von Lindens unübersehbar war. Die spektakulären Hochverratsprozesse von 1851/1852 mit ihren exorbitanten Strafen besonders für

114 Dies gilt insbesondere für Pfizer, dessen „Briefwechsel zweier Deutscher“ von 1831 mit seiner Polemik gegen die klein- und mittelstaatlichen Monarchen und der Betonung einer preußischen Mission in Deutschland Wilhelm empörte.

115 Sie alle besaßen nicht das Vertrauen des Königs. Vgl. dazu etwa Franz Mögle-HofACKer, Zur Entwicklung des Parlamentarismus in Württemberg. Der „Parlamentarismus der Krone" unter König Wilhelm I., Stuttgart 1981, S. 56, 58.

116 Vgl. etwa Wolfgang Schieder, 1848/49: Die ungewollte Revoluton, in: Carola Stern/ Heinrich August WinkLer (Hg.), Wendepunkte deutscher Geschichte 1848-1945, Frankfurt a. M. 1979, S.13-35, hier S.35. - Der „Beobachter“ konstatierte im Frühjahr den drobenden Ausbruch der großen Fürstenverschwörung; Nr. 87 vom 13.4.1849.

117 Otto Elben, Lebenserinnerungen 1823-1899, Stuttgart 1931, S.116. 
die Geflüchteten ${ }^{118}$ symbolisieren nicht nur diesen Reaktionskurs, sondern auch das grandiose Scheitern von Liberalen und Demokraten.

Es war wiederum Gottlieb Rau, der die kommende Entwicklung schon frühzeitig scharf und ahnungsvoll kritisierte. In seiner Gaildorfer Rede vom 12.3.1848 warnte er vor dem Trugschluss, den tiefen Krebsschaden der geistigen und materiellen Verkümmerung des Volkes jetzt noch mit Pressfreibeit, Schwurgerichten, Volksbewaffnung, deutschem Parlament und ähnlichen Maaßregeln alle in begegnen zu können ${ }^{119}$. Nach einer gescheiterten Landtagsbewerbung beschritt er den revolutionären Weg und scheiterte. Für dieses aus seiner Sicht konsequente Verhalten hatte er dann einen hohen Preis - Inhaftierung und Verbannung - zu zahlen ${ }^{120}$.

Carl Mayer, 1848/1849 einer der Führer der württembergischen Demokraten, wurde nach der Auflösung des Stuttgarter Rumpfparlaments 1849 ebenfalls zum gescheiterten Revolutionär ${ }^{121}$. Er zog nach langem Exil in der Schweiz, das er ungebrochen überstand, ein selbstkritisches Fazit - man sei „1848 in der Halbheit stecken geblieben" ${ }^{122}$.

118 Dazu Hans Maier, Die Hochverratsprozesse gegen Gottlieb Rau und August Becher nach der Revolution von 1848 in Württemberg, Pfaffenweiler 1992. - Der 1849 in die Schweiz geflüchtete „Beobachter“-Redakteur Weisser wurde in absentia zu 18 Jahren Zuchthaus verurteilt (Haller Tagblatt Nr. 31 vom 9.2.1852) und kehrte wohl 1863 als gebrochener und kranker Mann nach Württemberg zurück (Beob. Nr. 236 vom 10.10.1863). Er darf als eines der bedauernswertesten Opfer juristisch kaschierter staatlicher Willkür gelten.

119 Beob. Nr.17. vom 19.3.1848.

120 Dazu Eichele (wie Anm. 14).

$121 \mathrm{Zu}$ ihm Müllek, Carl Mayer (wie Anm. 106).

122 Zit. nach ebd., S. 45. Die Studie schildert u. a. ausführlich das Handeln und Lavieren der Demokraten sozusagen zwischen Römertreue und Revolution während der Revolutionsjahre. 